Research Paper

\title{
Less Invasive Endometrial Cancer Surgery with Extraperitoneal Pelvic and Para-aortic Lymphadenectomy via a Small Midline Abdominal Incision and the Retroperitoneal Approach
}

Shinichi Komiyama ${ }^{凶}$, Chiaki Takeya, Rena Takahashi, Sumito Nagasaki, Kaneyuki Kubushiro

Department of Gynecology, Toho University Ohashi Medical Center.

$\triangle$ Corresponding author: Shinichi Komiyama, MD, PhD. Associate Professor, Department of Gynecology, Toho University Ohashi Medical Center, 2-17-6, Ohashi, Meguro-ku, Tokyo 153-8582, Japan. TEL: +81-3-3468-1251 FAX: +81-3-3468-7795 Email: shinkomiyama@gmail.com.

(C) Ivyspring International Publisher. Reproduction is permitted for personal, noncommercial use, provided that the article is in whole, unmodified, and properly cited. See http://ivyspring.com/terms for terms and conditions.

Received: 2016.01.16; Accepted: 2016.03.15; Published: 2016.04.29

\begin{abstract}
[Objective] To achieve less invasive lymphadenectomy in endometrial cancer patients, we performed extraperitoneal pelvic and para-aortic lymphadenectomy via a small midline abdominal incision with retroperitoneal approach. The feasibility and safety of this method were investigated.

[Methods] Inclusion criteria were 1) endometrioid adenocarcinoma diagnosed by preoperative biopsy, 2) myometrial invasion by magnetic resonance imaging, and 3) no peritoneal dissemination or distant metastasis by computed tomography. Systematic extraperitoneal dissection of pelvic and para-aortic lymph nodes was performed via an approximately $12-\mathrm{cm}$ midline lower abdominal incision, after which hysterectomy and bilateral salpingo-oophorectomy were done (extraperitoneal group). The historical control group was patients who underwent standard transperitoneal lymphadenectomy followed by hysterectomy and bilateral salpingo-oophorectomy. The two groups were compared for demographic characteristics, perioperative factors, and complications.

[Results] A total of 62 patients were enrolled. Demographic and clinicopathological factors showed no differences between the extraperitoneal group $(n=34)$ and the historical control group $(n=28)$. The median number of pelvic ( 30 vs. 28 ) and para-aortic $(14$ vs. 17$)$ nodes dissected was also similar. However, median intraoperative blood loss was significantly smaller in the extraperitoneal group than the control group ( 220 vs. 573 g). Median operating time ( 265 vs. 323.5 $\mathrm{min}$ ), median laparotomy time ( $60 \mathrm{vs.} 295 \mathrm{~min}$ ), and median initial flatus time ( 8 vs. 32 hours) were all significantly shorter in the extraperitoneal group, while complications and severe postoperative pain were significantly less frequent.

[Conclusions] Our new technique was feasible, safe, and less invasive than standard laparotomy. It is an alternative to laparoscope-assisted or robotic procedures.
\end{abstract}

Key words: less invasive surgery, small midline abdominal incision, extraperitoneal pelvic and para-aortic lymphadenectomy, retroperitoneal approach, endometrial cancer.

\section{Introduction}

Endometrial cancer is the most common malignancy of the female genital tract in developed countries. In the USA, 52630 women were newly diagnosed with endometrial cancer in 2014 and 8590 women died of this disease in that year [1]. In Japan, 14763 women were newly diagnosed with endometrial cancer in 2011 and 2107 women died of it in 2013. Attention is being focused on endometrial 
cancer because its incidence and mortality rate are increasing [2]

Surgery is the standard treatment for endometrial cancer. Various surgical staging procedures can be added to the basic operation of hysterectomy and bilateral salpingo-oophorectomy, among which retroperitoneal lymphadenectomy is the most important because the International Federation of Gynecology and Obstetrics (FIGO) stage can be determined by pathological diagnosis and patients with a risk of postoperative recurrence can be identified and given appropriate therapy [3].

In the standard retroperitoneal lymphadenectomy procedure, pelvic and para-aortic lymphadenectomy are performed via the transperitoneal approach, traditionally. However, this is extremely invasive because of the long laparotomy time, large wound, and large intraperitoneal surgical field. It is also associated with various complications, such as heavy blood loss, paralytic ileus, deep venous thrombosis, lower limb lymphedema, severe wound pain, and prolonged hospitalization [4]. Accordingly, methods for reducing complications have been investigated, including minimally invasive surgery, such as laparoscope-assisted and robotic surgery.

Some gynecologic surgeons perform pelvic lymphadenectomy via the retroperitoneal approach during resection of uterine cervical cancer [5-6]. This technique is becoming an alternative to standard surgery because the short laparotomy time minimizes trauma to the intraperitoneal organs. We adopted this approach for pelvic lymphadenectomy in 2010. By extending it, we developed a new technique for simultaneous dissection of pelvic and para-aortic lymph nodes in the same surgical field through a small lower abdominal incision (abdominal extraperitoneal pelvic and para-aortic lymphadenectomy via the retroperitoneal approach), and we have used this method to treat endometrial cancer since 2011. The present study was performed to investigate the feasibility and safety of our retroperitoneal procedure for endometrial cancer by reviewing our experience to date.

\section{Materials and Methods}

\section{Eligibility criteria and preoperative evaluation}

Patients who met all of the following criteria were enrolled in the extraperitoneal group: preoperative biopsy revealed endometrioid adenocarcinoma (or suspected endometrioid adenocarcinoma); transvaginal ultrasonography and pelvic magnetic resonance image (MRI) detected an endometrial mass with myometrial invasion in the corpus but no infiltration of the cervical region or metastasis to the adnexae; thoracoabdominal computed tomography (CT) scans found no peritoneal dissemination, peritoneal carcinomatosis, or distant metastasis; the preoperative Eastern Cooperative Oncology Group (ECOG) performance status was $0-1$; standard investigations (hematology tests, biochemistry tests, coagulation tests, chest and abdominal X-ray films, and ECG) indicated adequate function of vital organs; and written consent to treatment by this surgical technique was obtained.

Patients were excluded if they met any of the following criteria: preoperative endometrial biopsy strongly suggested a diagnosis of serous adenocarcinoma, clear cell adenocarcinoma, carcinosarcoma, or sarcoma; a history of abdominal/pelvic radiotherapy; preoperative ultrasonography of lower limb veins revealed deep venous thrombosis; and the patient wanted to preserve fertility.

\section{Surgical technique}

Left pelvic and para-aortic lymphadenectomy via the retroperitoneal approach

Surgery is performed under general and epidural anesthesia. A 12-cm midline incision is made in the lower abdomen (Figure 1a), the subcutaneous tissue and anterior part of rectus abdominis are dissected, and the connective tissue space between the rectus abdominis sheath and the parietal peritoneum is developed toward the left inguinal region (Figure $1 b)$. The inferior epigastric vessels arising from the external iliac artery and vein and the round ligament are identified and transected near the pelvic wall (Figure 1c) to expose the external iliac artery and vein. The peritoneal sac containing intraperitoneal organs is separated from these vessels, after which the pelvic lymph node area is developed. Using an Octopus Retractor with a long hook, blunt digital dissection is performed to expose the common iliac artery up to the bifurcation of the abdominal aorta, and the peritoneal sac is displaced cranially. After the paravesical space, obturator nerve, ureter, and lateral umbilical ligament are identified, left pelvic lymphadenectomy is performed using a vessel sealing system and an electric scalpel. Dissection of the external iliac nodes, external suprainguinal nodes, obturator nodes, internal iliac nodes, common iliac nodes and presacral nodes is done in this order (Figure 1d). Titanium vascular clips are applied to the distal marginal lymphatics of the external suprainguinal and obturator nodes.

Next, the space between the left transversus abdominis muscle and the peritoneum (i.e., the left transversalis fascia) is separated sharply to free the peritoneal sac. By using an Octopus Retractor, the sac 
is displaced cranially from the left common iliac artery along the left side of the aorta to develop the para-aortic area. The operating table is placed in the Trendelenburg position at approximately 10 degrees to stop the peritoneal sac moving caudally. After the inferior pole with Gerota's fascia of the left kidney is confirmed and the inferior mesenteric artery (IMA) is identified (Figure 1e), para-aortic lymphadenectomy is performed along the left side, anterior and posterior of the aorta with dissection of lymph nodes caudal and cranial to the IMA in this order using a vessel sealing system and an electric scalpel. Since the kidneys and renal vessels are shifted cranially in the
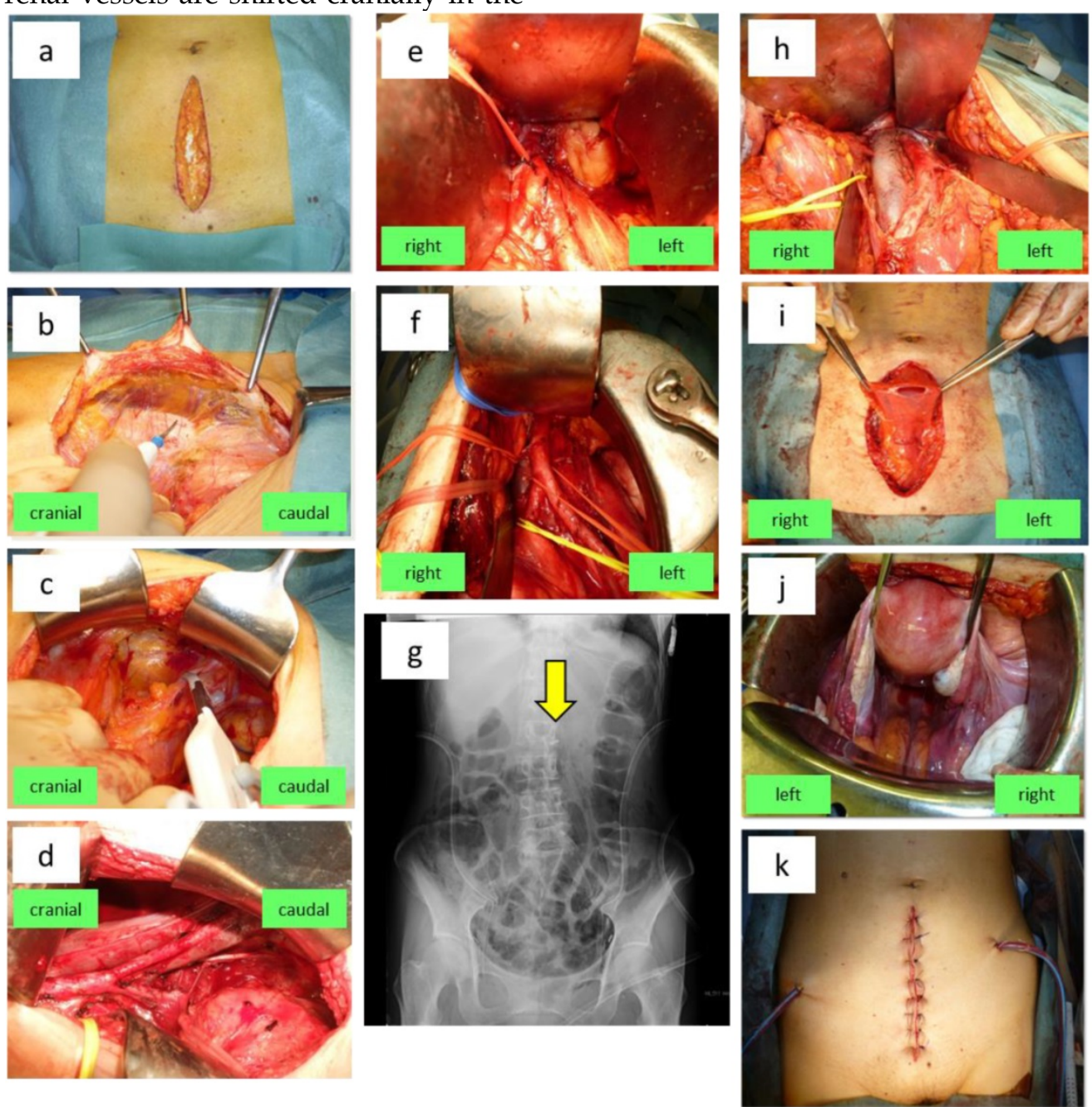

Figure 1. Abdominal extraperitoneal pelvic and para-aortic lymphadenectomy via the retroperitoneal approach followed by intraperitoneal total abdominal hysterectomy and bilateral salpingo-oophorectomy for endometrial cancer. (a) An approximately 12-cm incision is made in the lower abdomen. (b) The connective tissue space between the rectus abdominis sheath and the parietal peritoneum is developed toward the left inguinal region. (c) After the inferior epigastric vessels and the round ligament are identified, these structures are transected near the pelvic wall. (d) The peritoneal sac containing organs such as the intestinal tract is separated mainly from the external iliac artery and vein, and the pelvic lymph node area is developed. The paravesical space, obturator nerve, ureter (yellow vascular tape), and lateral umbilical ligament (white vascular tape) are identified. The external iliac, external suprainguinal, obturator, internal iliac, common iliac nodes and presacral nodes are dissected in this order. (e) The left transversalis fascia is separated, and the peritoneal sac containing the intraperitoneal organs is freed. Using an Octopus Retractor with a long hook, the sac is displaced from the common iliac artery in the cranial direction along the left side of the aorta to develop the para-aortic area. The inferior mesenteric artery (IMA) (red vascular tape) is identified. ( $f$ ) The para-aortic lymph nodes to the left side, anterior and posterior of the aorta are dissected in the order of nodes caudal to the IMA followed by nodes cranial to the IMA. (g) Dissection of para-aortic lymph nodes up to the level of L2 is confirmed by the position of the vascular clip on a postoperative plain abdominal X-ray film (arrow). (h) Para-aortic lymph nodes to the right of the inferior vena cava and nodes lying between the vena cava and aorta are dissected in the order of nodes caudal to the IMA followed by nodes cranial to the IMA. (i) After lymphadenectomy is completed, a midline incision is made in the peritoneum to enter the peritoneal cavity. (j) The uterus and bilateral adnexae are dissected via the extrafascial method. ( $k$ ) The midline peritoneal incision is closed with sutures. Then a retroperitoneal drain is placed, the wound is closed, and surgery is completed. 
Right pelvic and para-aortic lymphadenectomy via the retroperitoneal approach

Right pelvic lymphadenectomy is performed according to the procedure mentioned above. The transversalis fascia is separated sharply to free the peritoneal sac. Then, using an Octopus Retractor, the sac is displaced cranially from the right common iliac artery along the right side of the inferior vena cava. With the patient in the Trendelenburg position, the inferior pole of the right kidney and the IMA are confirmed. Then the para-aortic lymph nodes to the right, anterior and posterior of the inferior vena cava and the nodes between the vena cava and aorta are dissected in the order of nodes caudal to the IMA followed by those cranial to the IMA (Figure 1h). The operating table is returned to horizontal immediately after completing lymphadenectomy.

\section{Intraperitoneal total hysterectomy and bilateral salpingo-oophorectomy}

After completing lymph node dissection, a midline incision is made in the peritoneum and the peritoneal cavity is opened (Figure 1i). Careful observation is performed to detect tumor dissemination or involvement of the greater omentum, followed by peritoneal cytology. Next, using the extrafascial technique of dissecting the parametrium outside the uterine cervical fascia, the uterus and bilateral adnexae are removed together with a 5-7 $\mathrm{mm}$ vaginal cuff including the fornix (Figure 1j). The margin of the vaginal cuff and the posterior peritoneum in this area are sutured, while the posterior peritoneum is left open near the external iliac vessels and the site of transecting the infundibulopelvic ligament to allow inflow of retroperitoneal lymph into the peritoneal cavity. After an absorbable adhesion barrier is attached immediately beneath the wound, the midline peritoneal incision is sutured. Vacuum-assisted closure drains are placed in the bilateral paravesical spaces via the extraperitoneal approach and fixed to the abdominal wall. The rectus abdominis fascia and subcutaneous tissue are approximated in this order with absorbable thread, and surgery is completed by closing the skin with nylon thread or a stapler (Figure $1 \mathrm{k})$. In obese patients, a small-bore open silicone drain is fixed subcutaneously in the wound to prevent infection.

\section{Postoperative management}

A broad-spectrum penicillin derivative is administered intravenously during surgery and until postoperative day 2 for prophylaxis. Oral intake is started after confirming initial passage of flatus. The urethral catheter is removed 1 day after surgery and ambulation is commenced. Continuous opioid infusion is provided via an epidural catheter until 48 hours postoperatively. After confirming that there is no risk of postoperative hemorrhage, unfractionated heparin (5000 units) is administered subcutaneously every 12 hours from postoperative day 1 to day 3 to prevent venous thromboembolism. In addition, intermittent pneumatic compression of the lower limbs is performed during and after surgery, but is stopped immediately after the start of walking. The retroperitoneal drains are removed when unilateral flow decreases to approximately $100-150 \mathrm{~mL} /$ day. All patients are discharged within a few days after removal of the peritoneal drains.

\section{Evaluation and comparison}

Endpoints of this study were surgical feasibility and safety. The feasibility was evaluated according to perioperative factors, which contained intraoperative blood loss, total operating time, laparotomy time, lymphadenectomy time, number of dissected lymph nodes, time until initial passage of flatus, and time until removal of all drains (surrogate day of hospital discharge). The safety was investigated according to intraoperative and postoperative complications. Demographic characteristics and perioperative factors were compared between the two groups. Pain was evaluated at 48 hours after surgery. Pain intensity was assessed by using a five-item verbal rating scale (VRS, with 0 no pain, 1 mild pain, 2 moderate pain, 3 severe pain, and 4 extreme pain) [7]. Postoperative complications were classified as early $(<2$ weeks after surgery) or late (2 weeks to 3 months after surgery). Both intraoperative and postoperative complications were evaluated according to Common Terminology Criteria for Adverse Events (CTCAE) Version 4.0 [8].

Follow-up was performed every month from 4 weeks after surgery, including internal gynecological examination, transvaginal ultrasonography, hematology tests, biochemistry tests, and urinalysis at each visit. Vaginal cytology was performed every 6 months, while thoracoabdominal CT scanning was done once a year or when routine tests revealed an abnormality for investigation.

Among patients with endometrial cancer who underwent standard transperitoneal pelvic and para-aortic lymphadenectomy followed by intraperitoneal total abdominal hysterectomy and bilateral salpingo-oophorectomy during the 3-year period before 2010, those meeting the same inclusion criteria as the extraperitoneal group were enrolled as a historical control group. In this group, the vessel sealing system and vascular clips were not employed, but postoperative management was the same as in the 
extraperitoneal group.

All operations in both groups were performed by the same surgeon (S.K.). The study was approved by our institutional review board.

\section{Statistical analysis}

Perioperative factors were compared between the two groups by the Mann-Whitney U-test, while intraoperative and postoperative complications were compared by Fisher's exact test. Differences of $p<0.05$ were considered statistically significant. Statistical analysis was performed using GraphPad Prism version 6.0 for Windows (GraphPad Software, San Diego, CA).

\section{Results}

Among 62 patients enrolled, 34 patients underwent extraperitoneal pelvic and para-aortic lymphadenectomy via the retroperitoneal approach combined with intraabdominal total hysterectomy and bilateral salpingo-oophorectomy between January 2011 and January 2014 (extraperitoneal group), while 28 patients underwent standard transperitoneal pelvic and para-aortic lymphadenectomy followed by total hysterectomy and bilateral salpingo-oophorectomy between January 2008 and December 2010 (historical control group).

Table 1 displays the demographic characteristics of the two groups. Median age and median body mass index (BMI) showed no appreciable differences. In both groups, the FIGO stage was IA in approximately $60 \%$ and lymphovascular space invasion (LVSI) in approximately $40 \%$, IB in approximately $20 \%$, and IIIC with retroperitoneal lymph node metastasis in approximately $15 \%$. There were no patients in stages II or IIIB. With regard to tumor histology, so-called type 1 cancer accounted for nearly $80 \%$ and type 2 cancer for approximately $20 \%$ in both groups. Postoperative chemotherapy was given to stage IA patients with LVSI and patients in stage IB or higher, and approximately $60 \%$ of patients received chemotherapy in both groups. Radiotherapy was not performed in either group.

Table 2 summarizes the perioperative factors. Median intraoperative blood loss (range) was $220 \mathrm{~g}$ (41-1100) in the extraperitoneal group and $573 \mathrm{~g}$ $(268-2450)$ in the historical control group $(\mathrm{p}<0.001)$. Median total operating time was 265 min (198-370) and $323.5 \mathrm{~min}$ (238-424), median laparotomy time was $60 \mathrm{~min}$ (41-120) and $295 \mathrm{~min}$ (208-380), and median lymphadenectomy time was $178.5 \mathrm{~min}$ (139-280) and 235 min (160-330), respectively. These times were all significantly shorter in the extraperitoneal group. The median number of pelvic lymph nodes dissected was
30 (20-63) and 28 (21-42), while the median number of para-aortic lymph nodes dissected was 14 (4-37) and 17 (9-28), respectively. Median time until initial passage of flatus was 8 (3-20) and 32 hours (19-48) and median time until removal of all drains was 7.5 (4-18) and 20 days (14-30), respectively, both being significantly shorter in the extraperitoneal group $(\mathrm{p}<0.001)$.

Table 1. Demographic characteristics.

\begin{tabular}{|c|c|c|c|c|}
\hline & \multicolumn{2}{|c|}{ Extraperitoneal Group } & \multicolumn{2}{|c|}{ Historical Control Group } \\
\hline & \multicolumn{2}{|c|}{$\mathrm{n}=34$} & \multicolumn{2}{|c|}{$\mathrm{n}=28$} \\
\hline age (range) & \multicolumn{2}{|c|}{$55(37-78)$} & \multicolumn{2}{|c|}{$56(34-75)$} \\
\hline \multirow[t]{2}{*}{ BMI (range) } & \multicolumn{2}{|c|}{$22.6(19.1-30.3)$} & \multicolumn{2}{|c|}{$22.3(18.3-25.7)$} \\
\hline & $\mathrm{n}$ & $\%$ & $\mathrm{n}$ & $\%$ \\
\hline \multicolumn{5}{|l|}{ FIGO Stage } \\
\hline I A & 20 & 58.8 & 17 & 60.7 \\
\hline I B & 7 & 20.5 & 6 & 21.4 \\
\hline III A & 2 & 5.9 & 1 & 3.6 \\
\hline III C1 & 3 & 8.9 & 3 & 10.7 \\
\hline III C2 & 2 & 5.9 & 1 & 3.6 \\
\hline \multicolumn{5}{|c|}{ I A with LVSI * } \\
\hline negative & 14 & 58.9 & 10 & 64.3 \\
\hline positive & 6 & 41.1 & 7 & 35.7 \\
\hline \multicolumn{5}{|l|}{ Histology ** } \\
\hline EM G1 & 19 & 55.9 & 15 & 53.6 \\
\hline EM G2 & 8 & 23.5 & 6 & 21.4 \\
\hline EM G3 & $4^{* * *}$ & 11.8 & 4 & 14.3 \\
\hline serous & 2 & 5.9 & 2 & 7.1 \\
\hline others & $1^{* * * *}$ & 2.9 & $1^{* * * * *}$ & 3.6 \\
\hline \multicolumn{5}{|c|}{ Chemotherapy } \\
\hline yes & 20 & 58.8 & 18 & 64.2 \\
\hline no & 14 & 41.2 & 10 & 35.8 \\
\hline \multicolumn{5}{|l|}{ Radiotherapy } \\
\hline yes & 0 & & 0 & \\
\hline no & 34 & 100 & 28 & 100 \\
\hline
\end{tabular}

Table 2. Perioperative factors.

\begin{tabular}{|c|c|c|c|c|c|}
\hline & \multicolumn{2}{|c|}{$\begin{array}{l}\text { Extraperitoneal } \\
\text { Group }\end{array}$} & \multicolumn{2}{|c|}{$\begin{array}{l}\text { Historical Control } \\
\text { Group }\end{array}$} & \multirow[t]{2}{*}{$\mathrm{p}^{*}$} \\
\hline & median & range & median & range & \\
\hline $\begin{array}{l}\text { intraoperative blood loss } \\
(\mathrm{ml})\end{array}$ & 220 & $41-1100$ & 573 & $268-2450$ & $<0.001$ \\
\hline total operating time (min) & 265 & $198-370$ & 323.5 & $238-424$ & $<0.001$ \\
\hline laparotomy time (min) & 60 & $41-120$ & 295 & $208-380$ & $<0.001$ \\
\hline $\begin{array}{l}\text { lymphadenectomy time } \\
\text { (min) }\end{array}$ & 178.5 & $139-280$ & 235 & $160-330$ & 0.0164 \\
\hline number of dissected PLN ** & 30 & $20-63$ & 28 & $21-42$ & 0.2509 \\
\hline number of dissected PAN *** & 14 & $4-37$ & 17 & $(9-28)$ & 0.2385 \\
\hline $\begin{array}{l}\text { time until initial passage of } \\
\text { flatus }(\mathrm{hr})\end{array}$ & 8 & $(3-20)$ & 32 & $19-48$ & $<0.001$ \\
\hline $\begin{array}{l}\text { time until removal of all } \\
\text { drains (dav) }\end{array}$ & 7.5 & $(4-18)$ & 20 & $(14-30)$ & $<0.001$ \\
\hline
\end{tabular}

* Mann-Whitney's U test; ** PLN : pelvic lymph nodes; *** PAN : para-aortic lymph nodes 
Table 3 shows intraoperative and postoperative complications. Blood transfusion (excluding autotransfusion) was required by 1 patient $(2.9 \%)$ and 5 patients $(17.8 \%)$ in the extraperitoneal and control groups, respectively. Grade 1 venous injury was noted $1(2.9 \%)$ and $3(10.7 \%)$ patients, respectively. Urinary tract injury, gastrointestinal injury and neurological injury did not occur in the extraperitoneal group, while there was grade 1 ureteral injury and grade 1 obturator nerve injury in 1 control patient (3.6\%) each. None of these complications showed a significant difference between the two groups.

Table 3. Complications.

\begin{tabular}{|c|c|c|c|c|c|}
\hline & \multicolumn{2}{|c|}{$\begin{array}{l}\text { Extraperitoneal } \\
\text { Group }\end{array}$} & \multicolumn{3}{|c|}{$\begin{array}{l}\text { Historical Control } \mathrm{p}^{*} \\
\text { Group }\end{array}$} \\
\hline & $\mathrm{n}$ & $\%$ & $\mathrm{n}$ & $\%$ & \\
\hline \multicolumn{6}{|l|}{ Intraoperative } \\
\hline Blood transfusion ** & 1 & 2.9 & 5 & 17.8 & 0.0824 \\
\hline Venous injury & 1 & 2.9 & 3 & 10.7 & 0.3195 \\
\hline Urinary tract injury & 0 & & 1 & 3.6 & 0.4516 \\
\hline Gastrointestinal injury & 0 & & 0 & & \\
\hline Neurological injury & 0 & & 1 & 3.6 & 0.4516 \\
\hline \multicolumn{6}{|l|}{ Early postoperative ${ }^{* * *}$} \\
\hline Anemia & 1 & 2.9 & 5 & 17.8 & 0.0824 \\
\hline Hypoalbuminemia & 2 & 5.9 & 6 & 21.4 & 0.1253 \\
\hline Wound dehiscence & 1 & 2.9 & 3 & 10.7 & 0.3195 \\
\hline Wound infection & 2 & 5.9 & 4 & 14.3 & 0.3959 \\
\hline Lymphocele & 1 & 2.9 & 3 & 10.7 & 0.3195 \\
\hline Urinary tract infection & 1 & 2.9 & 2 & 7.1 & 0.5847 \\
\hline Ileus & 0 & & 2 & 7.1 & 0.1999 \\
\hline Venous thrombosis & 0 & & 0 & & \\
\hline Urinary fistula & 0 & & 0 & & \\
\hline Postoperative pain ${ }^{* * * *}$ & 0 & & 5 & 17.8 & 0.0152 \\
\hline \multicolumn{6}{|l|}{ Late postoperative ${ }^{* * * * *}$} \\
\hline Lower limb lymphedema & 1 & 2.9 & 8 & 30.8 & 0.0081 \\
\hline $\begin{array}{l}\text { Lower abdominal } \\
\text { lymphedema }\end{array}$ & 7 & 20.6 & 2 & 7.1 & 0.1662 \\
\hline Lymphocele & 5 & 14.7 & 4 & 14.3 & 0.6259 \\
\hline Ileus & 0 & & 1 & 3.6 & 0.4516 \\
\hline $\begin{array}{l}\text { All grade } 3 \text { or worse } \\
\text { complications }\end{array}$ & 1 & 5.9 & 7 & 25.0 & 0.0144 \\
\hline
\end{tabular}

As early postoperative complications, grade 2 or worse anemia was detected in 1 patient $(2.9 \%)$ from the extraperitoneal group and 5 patients $(17.8 \%)$ from the control group, while there was grade 2 or worse hypoalbuminemia in $2(5.9 \%)$ and $6(21.4 \%)$ patients, grade 1 wound dehiscence in 1 (2.9\%) and $3(10.7 \%)$ patients, grade 2 wound infection in $2(5.9 \%)$ and 4 $(14.3 \%)$ patients, grade 2 or worse lymphocele in 1 $(2.9 \%)$ and $3(10.7 \%)$ patients, and grade 3 urinary tract infection in $1(2.9 \%)$ and $2(7.1 \%)$ patients, respectively. Ileus did not occur in the extraperitoneal group, while 2 control patients $(7.1 \%)$ had grade 2 ileus. The incidence of these complications showed no significant differences between the two groups. However, severe postoperative pain (VRS $\geqq 3$ at 48 hours) did not occur in the extraperitoneal group, but was noted in 5 control patients $(17.8 \%)(p=0.0152)$.

Among late postoperative complications, grade 2 or worse lower limb lymphedema was significantly less frequent in the extraperitoneal group than the control group (1 patient $(2.9 \%)$ vs. 8 patients $(30.8 \%)$, $\mathrm{p}=0.0081$ ). However, grade 2 or worse lymphedema of the lower abdomen was more frequent in the extraperitoneal group (7 patients $(20.6 \%)$ vs. 2 patients $(7.1 \%))$, while grade 1 lymphocele was noted in 5 $(14.7 \%)$ and 4 patients $(14.3 \%)$, respectively. Ileus did not occur in the extraperitoneal group, while 1 patient (3.6\%) from the control group had grade 2 ileus. These complications showed no significant differences between the two groups. However, the incidence of all grade 3 or worse complications throughout the follow-up period was significantly lower in the extraperitoneal group ( $2.9 \%$ versus $25.0 \%, \mathrm{p}=0.0144$ ).

In the extraperitoneal group, all 34 patients are alive without recurrence after a median follow-up period (range) of 30 months (16-50). In the control group, 4 of the 28 patients $(14.2 \%)$ showed relapse during a median follow-up period (range) of 52 months (35-63). Two of these patients died of endometrial cancer at 35 and 38 months after surgery, 1 is alive with disease, and 1 is alive without disease after complete remission was induced by chemotherapy.

\section{Discussion}

Retroperitoneal lymphadenectomy is useful for both diagnosis and treatment of endometrial cancer. Because lymphadenectomy determines whether or not a patient has lymph node metastasis, patients requiring postoperative therapy can be identified and unnecessary postoperative therapy can be avoided, while any existing metastases are removed [9]. However, retroperitoneal lymphadenectomy is highly invasive, particularly when both the pelvic and para-aortic lymph nodes are dissected systematically, and such extensive lymphadenectomy can have disadvantages for patients. Therefore, individualized lymphadenectomy has been recommended recently [9]. According to the NCCN Guidelines ${ }^{\circledR}$, the external iliac, internal iliac, obturator, and common iliac nodes should be dissected in this order in some patients with endometrial cancer localized to the uterus. It is also recommended that the para-aortic lymph nodes should be dissected up to the renal vessels in patients with deep myometrial invasion or highly malignant histology [3]. 
Retroperitoneal lymph node metastasis has been reported in $12-16 \%$ of patients with endometrial cancer, with involvement being in the order of para-aortic nodes $(9.8 \%)$, obturator nodes $(9.4 \%)$, internal iliac nodes $(7.1 \%)$, common iliac nodes $(5.6 \%)$ [10-11]. It has also been reported that after excluding so-called low-risk cases, $17 \%$ of patients have retroperitoneal lymph node metastasis to pelvic lymph nodes and para-aortic lymph nodes are involved in $12 \%$, with more than half of para-aortic metastases being to the high para-aortic nodes lying between the IMA and the renal artery [12]. Therefore, it is important to accurately separate the approximately $20 \%$ of patients who require lymphadenectomy from the $80 \%$ who do not need it to avoid overtreatment and undertreatment. It is also very important to appropriately manage the approximately $10 \%$ of patients requiring para-aortic lymphadenectomy.

Various predictors of lymph node metastasis and treatment algorithms have been investigated in attempt to efficiently identify patients requiring retroperitoneal lymphadenectomy [9,13-14]. In the Europe, it is considered that retroperitoneal lymphadenectomy should not be performed during initial surgery for endometrial cancer that is preoperatively evaluated as low-risk or intermediate-risk, and two-stage lymphadenectomy based on postoperative diagnosis is recommended [15].

However, it is extremely difficult to diagnose retroperitoneal lymph node metastasis and determine risk factors preoperatively, including important factors such as histological differentiation, myometrial invasion, and lymphovascular space invasion. It has been reported that the surgical stage was higher in $27.4 \%$ of patients because lymph node metastasis was diagnosed histopathologically and addition of retroperitoneal lymphadenectomy was considered to be necessary in $21.0 \%$ based on evaluation after initial surgery [16]. Thus, both overtreatment and undertreatment are likely to occur if appropriate lymphadenectomy is not done. It has also been reported that the two-stage surgery recommended in Europe is rarely performed in actual clinical practice [16].

There have been few large-scale prospective clinical studies concerning the usefulness of systematic lymphadenectomy for early endometrial cancer. Among them, two prospective randomized studies found that pelvic lymphadenectomy was not useful [17-18]. However, there were significant problems with both studies (many patients in both groups received postoperative radiotherapy, many low-risk patients were included, and surgical quality was problematic in the ASTEC trial), so the utility of lymphadenectomy cannot be ruled out by these results. To date, there have been no prospective randomized comparative investigations of systematic para-aortic lymphadenectomy.

On the other hand, a well-designed large-scale retrospective cohort study (the SEPAL trial) has been performed to assess para-aortic lymphadenectomy [19]. This study showed that systematic dissection of both pelvic and para-aortic lymph nodes significantly improved progression-free survival and overall survival in intermediate-risk and high-risk patients. Also, a meta-analysis of retrospective studies and randomized comparative studies revealed that systematic dissection of 10 or more pelvic and para-aortic lymph nodes improves overall survival in intermediate -risk/high-risk patients [20], while another retrospective study demonstrated that efficacy increases with an increase of dissected lymph nodes [21-22]. In patients with endometrial cancer, it has been reported that at least 22 pelvic lymph nodes, or at least 10 para-aortic lymph nodes should be dissected for diagnosis [23]. These reports suggest that we should avoid excessively narrowing the extent of lymphadenectomy or reducing the number of nodes dissected.

Postoperative adjuvant therapy for endometrial cancer is not standardized, and the influence of surgical invasion varies considerably depending on whether radiotherapy or chemotherapy is given. According to recent surveys, management of endometrial cancer varies greatly between countries, with systematic pelvic and para-aortic lymphadenectomy being favored in Europe and Japan, while biopsy is frequent in the USA and UK. Para-aortic lymphadenectomy is performed up to the renal artery level at $80 \%$ or more of institutions in Europe and Japan, while the corresponding percentage is only $51 \%$ in the USA and UK. [24]. Also, postoperative therapy is usually systemic chemotherapy alone in Japan, while radiotherapy and chemotherapy are often combined in Western countries [24].

Our policy is to perform systematic pelvic and para-aortic lymphadenectomy followed by extrafascial total hysterectomy and bilateral salpingo-oophorectomy in all patients with endometrial cancer, except those who are considered to be low risk. If the risk is evaluated as intermediate to high after surgery, platinum-based chemotherapy is given. If preoperative endometrial biopsy strongly suggests serous adenocarcinoma, clear cell adenocarcinoma, carcinosarcoma, or sarcoma, we avoid surgery via a small lower abdominal incision because of the high risk of the tumor involving a wide 
intraperitoneal area including the epigastric region. Instead, the peritoneal cavity is explored adequately via a longer abdominal incision, and omentectomy is performed along with pelvic and para-aortic lymphadenectomy.

In recent years, laparoscope-assisted and robotic surgery have been performed to reduce the invasiveness of surgery for endometrial cancer [25-26]. These procedures can decrease the wound size, blood loss, postoperative complications, postoperative pain, and duration of hospitalization. However, there are also disadvantages, such as a very long operating time, dissection of fewer lymph nodes, low feasibility in elderly or obese patients, frequent intraoperative complications, frequent conversion to open laparotomy, and requirement for considerable training [27-28]. In addition, expensive devices with high maintenance costs are needed, resulting in an increase of medical costs [29]. Therefore, although the number of gynecological oncologists selecting laparoscope-assisted and robotic surgery has increased, many surgeons still perform standard laparotomy as first-line treatment even in developed countries [30].

Systematic dissection of both pelvic and para-aortic lymph nodes via a small midline abdominal incision and the retroperitoneal approach without laparoscopic or robotic assistance has not been reported before as radical surgery for gynecological malignancies, including endometrial cancer. This technique has not been widely adopted, presumably because recognition of the retroperitoneal approach is still low and because surgeons are often interested in the latest medical devices and techniques. Interestingly, urologists have performed extraperitoneal lymphadenectomy by a similar technique to ours and their data on the operating time, blood loss, number of dissected lymph nodes, and postoperative complications were also similar. However, their surgical technique differed from ours in that a long incision was made from the xiphisterum to the suprapubic region and intraperitoneal manipulation was not performed [31].

Our technique is less invasive than standard laparotomy with respect to wound size, operating time, intraoperative blood loss, and intraoperative/postoperative complications. In particular, pneumoperitoneum is not needed and the contact time with intraperitoneal organs such as the intestinal tract is very short, so intestinal peristalsis recovers soon after surgery and postoperative ileus does not occur. In this respect, our technique is clearly less invasive than laparoscope-assisted or robotic surgery. Furthermore, laparoscope-assisted or robotic surgery is not necessarily less invasive because it requires a longer operating time that may lead to problems related to prolonged general anesthesia or patients may develop increased intracranial and intraocular pressure due to pneumoperitoneum and positioning [32-33]. Moreover, our method employs similar surgical instruments to those for standard laparotomy and transperitoneal lymphadenectomy, and expensive devices like those used for laparoscope-assisted or robotic surgery are not required. Our technique achieves similar lymph node dissection to standard lymphadenectomy with a shorter operating time. It may also reduce intraoperative blood loss, although decreased blood loss was probably related to the vessel sealing system we adopted in addition to the retroperitoneal approach.

After retroperitoneal lymphadenectomy, approximately $10-60 \%$ and $3.4-5.8 \%$ of patients develop asymptomatic and symptomatic lymphocele, respectively [34]. Dissection of more lymph nodes, positive lymph node metastasis, and a shorter intrapelvic drainage time are related to postoperative occurrence of lymphocele and lymphedema [35]. With our technique, the incidence of asymptomatic/ symptomatic lymphocele was lower than reported previously and the incidence of lower limb lymphedema was also significantly lower (13-38\%) [36]. The incidence of lymphocele and lower limb lymphedema was probably reduced by multiple factors, such as not suturing the posterior peritoneum, drain placement, ligation of lymphatics with vascular clips, and sealing of lymphatics with a vessel sealing system. However, infection and patient discomfort are increased by longer use of a drain tube, along with prolonged hospitalization and increased medical costs [37], and another study found that pelvic drainage did not inhibit the occurrence of lymphocele after lymphadenectomy [38]. On the other hands, our method was associated with a higher incidence of lower abdominal lymphedema affecting the pubic, inguinal, and external genital regions, presumably due to development of the surgical field via the retroperitoneal approach. Among chronic complications, lymphedema not only impairs quality of life but affects the body image of female patients [36]. At present, we use Chinese herbal medicines to treat lower abdominal lymphedema after retroperitoneal lymphadenectomy [39].

\section{Conclusion}

The advantages of performing extraperitoneal pelvic and para-aortic lymphadenectomy followed by intraperitoneal total hysterectomy and bilateral salpingo-oophorectomy include a small wound, short laparotomy time, minimal intraperitoneal 
invasiveness, maintenance of intestinal peristalsis (no postoperative ileus), early postoperative oral intake, less postoperative pain, and less perioperative complications. In addition, this technique does not require expensive medical devices. Accordingly, it may be an alternative to laparoscope-assisted or robotic surgery. We will plan a randomized study to investigate the non-inferiority between laparoscopic or robotic procedure and our procedure in the future.

\section{Acknowledgments}

We would like to sincerely thank Professor Pierluigi Benedetti Panici ("Spapienza" University of Rome) for his demonstration of extraperitoneal abdominal radical hysterectomy that encouraged us to develop the surgical technique reported here.

This work was supported by JSPS KAKENHI Grant-in-Aid for Scientific Research (C) Grant Number 25462617.

\section{Competing Interests}

The authors have declared that no competing interest exists.

\section{References}

1. Siegel R, Ma J, Zou Z, Jemal A. Cancer statistics, 2014. CA Cancer J Clin 2014;64-29.

2. [Internet] Cancer Information Service, National Cancer Center, Japan. Cancer Registry and Statistics. http://ganjoho.jp/reg_stat/index.html

3. [Internet] NCCN Clinical Practice Guidelines in Oncology (NCCN Guidelines $\left.{ }^{\circledR}\right) \quad$ Uterine Neoplasms version 2. http://www.nccn.org/ professionals/physician_gls/pdf/uterine.pdf

4. Chi DS, Bristow RE, Cibula D. Surgical Principles in Gynecologic Oncology. In: Barakat R, Berchuck A, Markman M, Randall ME, eds. Principles and Practice of Gynecologic Oncology; 6th ed. Lippincott Williams \& Wilkins; 2013:190-209.

5. Savino L, Borruto F, Comparetto C, Massi GB. Radical vaginal hysterectomy with extraperitoneal pelvic lymphadenectomy in cervical cancer. Eur J Gynaecol Oncol 2001;22:31-5

6. Panici PB, Di Donato V, Plotti F, Musella A, Sansone M, Angioli R, Perniola G, Bellati F. Feasibility and safety of type $\mathrm{C} 2$ total extraperitoneal abdominal radical hysterectomy (TEARH) for locally advanced cervical cancer. Gynecol Oncol 2011;120:423-9.

7. Hjermstad MJ, Fayers PM, Haugen DF, Caraceni A, Hanks GW, Loge JH, Fainsinger $\mathrm{R}$, Aass N, Kaasa S; European Palliative Care Research Collaborative (EPCRC). Studies comparing Numerical Rating Scales, Verbal Rating Scales, and Visual Analogue Scales for assessment of pain intensity in adults: a systematic literature review. J Pain Symptom Manage 2011;41:1073-93.

8. [Internet] Common Terminology Criteria for Adverse Events (CTCAE) Version 4.0. http://evs.nci.nih.gov/ftp1/CTCAE/CTCAE_4.03_2010-0614_QuickReference 8.5x11.pdf

9. Bogani G, Dowdy SC, Cliby WA, Ghezzi F, Rossetti D, Mariani A. Role of pelvic and para-aortic lymphadenectomy in endometrial cancer: current evidence. J Obstet Gynaecol Res 2014;40:301-11.

10. Hiura M, Nogawa T, Matsumoto T, Yokoyama T, Shiroyama Y, Wroblewski J. Long-term survival in patients with para-aortic lymph node metastasis with systematic retroperitoneal lymphadenectomy followed by adjuvant chemotherapy in endometrial carcinoma. Int J Gynecol Cancer 2010;20:1000-5.

11. Odagiri T, Watari H, Kato T, Mitamura T, Hosaka M, Sudo S, Takeda M, Kobayashi N, Dong P, Todo Y, Kudo M, Sakuragi N. Distribution of lymph node metastasis sites in endometrial cancer undergoing systematic pelvic and para-aortic lymphadenectomy: a proposal of optimal lymphadenectomy for future clinical trials. Ann Surg Oncol 2014;21:2755-61.

12. Kumar S, Podratz KC, Bakkum-Gamez JN, Dowdy SC, Weaver AL, McGree ME, Cliby WA, Keeney GL, Thomas G, Mariani A. Prospective assessment of the prevalence of pelvic, paraaortic and high paraaortic lymph node metastasis in endometrial cancer. Gynecol Oncol 2014;132:38-43.

13. Mariani A, Sebo TJ, Katzmann JA, Keeney GL, Roche PC, Lesnick TG, Podratz KC. Pretreatment assessment of prognostic indicators in endometrial cancer. Am J Obstet Gynecol 2000;182:1535-44.
14. Todo Y, Choi HJ, Kang S, Kim JW, Nam JH, Watari H, Tamakoshi A, Sakuragi N. Clinical significance of tumor volume in endometrial cancer: a Japan-Korea cooperative study. Gynecol Oncol 2013;131:294-8.

15. L'Institut National du Cancer (INCa) Cancer de l'endomètre. Collection Recommandations \& référentiels. Boulogne-Billancourt: INCa; 2010.

16. Arsène $E$, Bleu $G$, Merlot $B$, Boulanger L, Vinatier D, Kerdraon $O$, Collinet $P$. Implications of a two-step procedure in surgical management of patients with early-stage endometrioid endometrial cancer. J Gynecol Oncol 2015;26:125-33.

17. Benedetti Panici P, Basile S, Maneschi F, Alberto Lissoni A, Signorelli M, Scambia G, Angioli R, Tateo S, Mangili G, Katsaros D, Garozzo G, Campagnutta E, Donadello N, Greggi S, Melpignano M, Raspagliesi F, Ragni N, Cormio G, Grassi R, Franchi M, Giannarelli D, Fossati R, Torri V, Amoroso $\mathrm{M}$, Crocè C, Mangioni C. Systematic pelvic lymphadenectomy vs. no lymphadenectomy in early-stage endometrial carcinoma: randomized clinical trial. J Natl Cancer Inst 2008;100:1707-16.

18. ASTEC study group, Kitchener H, Swart AM, Qian Q, Amos C, Parmar MK. Efficacy of systematic pelvic lymphadenectomy in endometrial cancer (MRC ASTEC trial): a randomised study. Lancet 2009;373:125-36.

19. Todo Y, Kato H, Kaneuchi M, Watari H, Takeda M, Sakuragi N. Survival effect of para-aortic lymphadenectomy in endometrial cancer (SEPAL study): a retrospective cohort analysis. Lancet 2010;375:1165-72.

20. Kim HS, Suh DH, Kim MK, Chung HH, Park NH, Song YS. Systematic lymphadenectomy for survival in patients with endometrial cancer: a meta-analysis. Jpn J Clin Oncol 2012;42:405-12.

21. Chan JK, Cheung MK, Huh WK, Osann K, Husain A, Teng NN, Kapp DS. Therapeutic role of lymph node resection in endometrioid corpus cancer: a study of 12,333 patients. Cancer 2006;107:1823-30.

22. Abu-Rustum NR, Iasonos A, Zhou Q, Oke E, Soslow RA, Alektiar KM, Chi DS, Barakat RR. Is there a therapeutic impact to regional lymphadenectomy in the surgical treatment of endometrial carcinoma? Am J Obstet Gynecol 2008;198:457.e1-5

23. Bakkum-Gamez JN, Mariani A, Dowdy SC, Weaver AL, McGree ME, Cliby WA, Gostout BS, Stanhope CR, Wilson TO, Podratz KC. The impact of surgical guidelines and periodic quality assessment on the staging of endometrial cancer. Gynecol Oncol 2011;123:58-64.

24. Fotopoulou C, Kraetschell R, Dowdy S, Fujiwara K, Yaegashi N, Larusso D, Casado A, Mahner S, Herzog TJ, Kehoe S, Vergote I, Miller DS, Marth C, Fujii $\mathrm{S}$, Sehouli J. Surgical and systemic management of endometrial cancer: an international survey. Arch Gynecol Obstet 2015;291:897-905

25. Walker JL, Piedmonte MR, Spirtos NM, Eisenkop SM, Schlaerth JB, Mannel RS, Spiegel G, Barakat R, Pearl ML, Sharma SK. Laparoscopy compared with laparotomy for comprehensive surgical staging of uterine cancer: Gynecologic Oncology Group Study LAP2. J Clin Oncol 2009;27:5331-6.

26. Cardenas-Goicoechea J, Shepherd A, Momeni M, Mandeli J, Chuang L, Gretz $\mathrm{H}$, Fishman D, Rahaman J, Randall T. Survival analysis of robotic versus traditional laparoscopic surgical staging for endometrial cancer. Am J Obstet Gynecol 2014;210:160.e1-160.e11.

27. Zullo F, Falbo A, Palomba S. Safety of laparoscopy vs laparotomy in the surgical staging of endometrial cancer: a systematic review and metaanalysis of randomized controlled trials. Am J Obstet Gynecol 2012;207:94-100.

28. Ran L, Jin J, Xu Y, Bu Y, Song F. Comparison of robotic surgery with laparoscopy and laparotomy for treatment of endometrial cancer: a meta-analysis. PLoS One 2014;9:e108361. doi: 10.1371/journal.pone.0108361. eCollection 2014

29. Tapper AM, Hannola M, Zeitlin R, Isojärvi J, Sintonen H, Ikonen TS, A systematic review and cost analysis of robot-assisted hysterectomy in malignant and benign conditions. Eur J Obstet Gynecol Reprod Biol 2014;177:1-10

30. Greggi S, Franchi M, Aletti G, Biglia N, Ditto A, Fagotti A, Giorda G, Mangili G, Odicino F, Salerno MG, Vizza E, Scaffa C, Scollo P. Management of endometrial cancer in Italy: a national survey endorsed by the Italian Society of Gynecologic Oncology. Int J Surg 2014;12:1038-44.

31. Kim P, Syan-Bhanvadia S, Djaladat H, Faber K, Tadros NN, Nichols C, Daneshmand S. Midline extraperitoneal approach for retroperitoneal lymph node dissection for testicular germ cell tumor. Urology 2012;80:941-5.

32. Weber ED, Colyer MH, Lesser RL, Subramanian PS. Posterior ischemic optic neuropathy after minimally invasive prostatectomy. J Neuroophthalmol 2007;27:285-7.

33. Park EY, Koo BN, Min KT, Nam SH. The effect of pneumoperitoneum in the steep Trendelenburg position on cerebral oxygenation. Acta Anaesthesiol Scand 2009;53:895-9.

34. Zikan M, Fischerova D, Pinkavova I, Slama J, Weinberger V, Dusek L, Cibula D. A prospective study examining the incidence of asymptomatic and symptomatic lymphoceles following lymphadenectomy in patients with gynecological cancer. Gynecol Oncol.2015;137:291-8.

35. van de Poll-Franse LV, Pijnenborg JM, Boll D, Vos MC, van den Berg $\mathrm{H}_{\text {, }}$ Lybeert ML, de Winter K, Kruitwagen RF. Health related quality of life and symptoms after pelvic lymphadenectomy or radiotherapy vs. no adjuvant regional treatment in early-stage endometrial carcinoma: a large population-based study. Gynecol Oncol 2012;127:153-60.

36. Beesley VL, Rowlands IJ, Hayes SC, Janda M, O'Rourke P, Marquart L, Quinn MA, Spurdle AB, Obermair A, Brand A, Oehler MK, Leung Y, McQuire L, Webb PM; Australian National Endometrial Cancer Study Group. Incidence, risk factors and estimates of a woman's risk of developing secondary lower 
limb lymphedema and lymphedema-specific supportive care needs in women treated for endometrial cancer. Gynecol Oncol 2015;136:87-93.

37. Dowdy SC, Borah BJ, Bakkum-Gamez JN, Kumar S, Weaver AL, McGree ME,

Haas LR, Cliby WA, Podratz KC. Factors predictive of postoperative morbidity and cost in patients with endometrial cancer. Obstet Gynecol 2012;120:1419-27.

38. Charoenkwan K, Kietpeerakool C. Retroperitoneal drainage versus no drainage after pelvic lymphadenectomy for the prevention of lymphocyst formation in patients with gynaecological malignancies. Cochrane Database Syst Rev 2014 Jun 4;6:CD007387.

39. Komiyama S, Takeya C, Takahashi R, Yamamoto Y, Kubushiro K. Feasibility study on the effectiveness of Goreisan-based Kampo therapy for lower abdominal lymphedema after retroperitoneal lymphadenectomy via extraperitoneal approach. J Obstet Gynaecol Res 2015;41:1449-56. 